\title{
List of upcoming conferences
}

American Contact Dermatitis Society $24^{\text {th }}$ Annual Meeting

February 28 2013: Miami beach, Florida, United States

E-mail: info@contactderm.org

Website: http://www.contactderm.org/

American Academy of Dermatology- $71^{\text {st }}$ Annual Meeting

March 1-5 2013: Miami Beach, Florida, United States

E-mail: MRC@aad.org

Website: http://www.aad.org

$3^{\text {rd }}$ International Conference on Clinical \& Experimental Dermatology

March 15-17 ${ }^{\text {th }}$ 2013: Hilton Chicago, Northbrook, USA

E-mail: dermatology2013@omicsgroup.co

Website: http:/www.omicsgroup.com/conferences/dermatology-2013/

Dubai Derma 2013- Dubai World Dermatology and Laser Conference and Exhibition

April 16-18 ${ }^{\text {th }}$ 2013: Dubai, United Arab Emirates

E-mail: triza.samson@index.ae

Website: www.dubaiderma.com

International Investigative Dermatology

8th-11th May 2013, Edinburgh, Scotland

Email: iid2013@bad.org.uk

Website: http://www.iid2013.org

Psoriasis 2013: $4^{\text {th }}$ Congress of the Psoriasis International Network

4-6 ${ }^{\text {th }}$ July 2013, Paris, France

Email:pso2013@mci-group.com

Website: http://www.pso2013.com/

Aesthetics 2013

23-25 ${ }^{\text {th }}$ August 2013, New Delhi, India

Website: www.aestheticsdelhi.com

$17^{\text {th }}$ Annual Congress The Cosmetic Dermatology Society (India)

Pre Congress Workshop: August 29-30 ${ }^{\text {th }} 2013$, Kochi, Kerala, India

Congress: August 30 ${ }^{\text {th }}$-September 1st 2013, Kochi, Kerala, India.

E-mail: cosderminidia@gmail.com,pravin.events@gmail.com

Website: http://www.cosdermindia.in/, www.cosdermindia2013.com

$22^{\text {nd }}$ EADV Congress

2-6 ${ }^{\text {th }}$ October 2013: Istanbul, Turkey

Website: http://www.eadv.org/

$11^{\text {th }}$ International Congress of Dermatology

$4^{\text {th }}-7^{\text {th }}$ December 2013: Greater Noida, Delhi NCR, India

Website: http:/www.icd2013.com/ 